\title{
In vacuum permanent magnet wiggler optimized for the production of hard $\mathrm{x}$ rays
}

\author{
O. Marcouille, ${ }^{*}$ N. Bechu, P. Berteaud, P. Brunelle, L. Chapuis, C. Herbeaux, A. Lestrade, J.-L. Marlats, \\ A. Mary, M. Massal, A. Nadji, K. Tavakoli, M. Valleau, J. Veteran, and M.-E. Couprie \\ Synchrotron SOLEIL, L'Orme des Merisiers, Bâtiment A, Saint-Aubin, Gif-sur-Yvette-91192, France
}

\author{
J.-M. Filhol
}

Fusion for Energy-C/Josep Pla 2-Torres Diagonal Litoral Edifice B3, Barcelona-08019, Spain

(Received 12 October 2012; published 16 May 2013)

\begin{abstract}
A new concept of wiggler has been designed and realized at SOLEIL to produce high energy photons in low/intermediate electron storage rings. Instead of using the superconducting technology which requires new equipment and instrumentation, heavy maintenance, and additional running costs, we have proposed to build a compact in-vacuum small gap short period wiggler that operates rather at moderate field than at high field. The wiggler composed of 38 periods of $50 \mathrm{~mm}$ produces $2.1 \mathrm{~T}$ at a gap of $5.5 \mathrm{~mm}$. The moderate value of the magnetic field enables one to limit the effects on the beam dynamics and to avoid excessive power and magnetic forces. In this purpose, the narrow magnetic system has been equipped with a counterforce device made of nonmagnetic springs. The roll-off resulting from the small size of poles has been compensated in situ by permanent magnet magic fingers. This paper reports the phases of design, construction, magnetic measurements, and on-beam tests of the in-vacuum wiggler WSV50.
\end{abstract}

DOI: 10.1103/PhysRevSTAB.16.050702

PACS numbers: 85.70.-w, 41.60.-m, 07.85.Qe

\section{INTRODUCTION}

Insertion devices (ID) are magnetic systems generating a spatial periodic magnetic field [1] which can be produced either by permanent magnets arrays, electromagnets, or by superconducting coils [2]. Because of the presence of the periodic field, electrons entering the ID wiggle and radiate an electromagnetic wave at the fundamental wavelength $\lambda_{r}$ and its harmonics $\lambda_{r} / n$, according to

$$
\lambda_{r}=\frac{\lambda_{0}}{2 \gamma^{2}}\left(1+\frac{a_{w}^{2}}{2}+\gamma^{2} \varphi^{2}\right),
$$

where $n$ is the harmonic number, $\lambda_{0}$ the magnetic period, $\gamma$ the relativistic factor, and $\varphi$ the observation angle. $a_{w}$ is the deflection parameter defined by

$$
a_{w}=\frac{e B_{0} \lambda_{0}}{2 \pi m c}
$$

where $e$ is the electron charge, $m$ the electron rest mass, $c$ the light velocity, and $B_{0}$ is the magnitude of the magnetic field. The $a_{w}$ parameter can be modified by changing either the magnetic field $B_{0}$ or the magnetic period $\lambda_{0}$ [3]. The radiated spectrum depends on the value of $a_{w}$. At low $a_{w}$ value, the ID operates in the so-called "undulator regime" with few radiated harmonics. At high $a_{w}$ value, in the wiggler regime, the number of harmonics increases and

\footnotetext{
*olivier.marcouille@synchrotron-soleil.fr
}

Published by the American Physical Society under the terms of the Creative Commons Attribution 3.0 License. Further distribution of this work must maintain attribution to the author(s) and the published article's title, journal citation, and DOI. they overlap each other at high photon energy [4] leading to a continuous spectrum similar to that produced by a pure bending magnet. Similarly to the bending magnet radiation, the critical energy $E_{c}$ of the ID operating in the wiggler regime is expressed as [4]

$$
E_{c}[\mathrm{keV}]=0.665 B_{0}[T] E_{e}^{2}[\mathrm{GeV}],
$$

where $E_{e}$ is the electron energy. The on-axis angular flux produced by the ID operating in the wiggler regime is written as [4]

$$
\begin{aligned}
{\left[\frac{d N}{d \Omega}\right]_{\mathrm{ID}} } & =2 \cdot \frac{L}{\lambda_{0}} \cdot\left[\frac{d N}{d \Omega}\right]_{\mathrm{BM}} \\
& =2.65 \times 10^{16} \frac{L}{\lambda_{0}} \times I \times E_{e}^{2} \times\left(\frac{E}{E_{c}}\right)^{2} \times\left[K_{2 / 3}\left(\frac{E}{2 E_{c}}\right)\right]^{2},
\end{aligned}
$$

where $I$ is the stored electron beam current, $L$ the ID length, $E$ the photon energy, and $K$ the modified Bessel function. The maximum of the angular flux is reached when the required photon energy is close to the critical energy. High energy photons are usually produced by high field wigglers using in particular superconducting technology. At BESSY II (1.9 GeV) [5] and SIBERIA II (2.5 GeV) [6], superconducting wigglers made of 17 and 21 poles generating magnetic fields of 7 and $7.5 \mathrm{~T}$ with periods of 148 and $164 \mathrm{~mm}$, respectively, are in operation. Even if they produce photons of high energy, high field wigglers generate a high power [7] which leads to damages on beam line optics and a dedicated front end is required. Electron beam dynamics is also impacted [8]. Tune shifts, energy spread, and horizontal emittance changes induced by the operation of IDs grow with the length of the ID and the 
field, and decrease with the square of the particle energy. The effects of the magnetic field on the beam lifetime get more and more pronounced as the particle energy gets lower. This is the reason it is preferable to build rather moderate or low magnetic field wigglers on intermediate energy storage rings.

Shortening the period of the ID is an alternative solution to decrease the magnetic field [9]. Although the angular flux produced by each radiating pole of the wiggler is lowered, the total flux is kept constant because the number of radiating pole increases. This enables one to produce similar flux with lower power. At DIAMOND (3 GeV) [10] and ELETTRA (2.1 GeV) [11] two superconducting wigglers of 49 poles producing a field of $3.5 \mathrm{~T}$ with periods of 60 and $64 \mathrm{~mm}$, respectively, have been tested on beam. At Canadian Light Source $(2.9 \mathrm{GeV})$ the superconducting wiggler operates with a low field and a small period $\left(B_{0}=2 \mathrm{~T}, \lambda_{0}=34 \mathrm{~mm}\right)$ [12], and to avoid the structure appearing in the spectrum because of the low $a_{w}$ value, the spatial periodicity of the magnetic field has been modulated.

However, the operation of a superconducting wiggler involves technical difficulties. Any excess of power deposited by the electron beam or synchrotron radiation results in quenches and damages. Liners, in particular, are the most frequently damaged components of the superconducting wigglers [13,14]. Superconducting wiggler operation needs also heavy maintenance such as regular replacement of cold head of cryocoolers, adsorbers of compressors, filling of $\mathrm{LN}_{2} / \mathrm{LHe}$ (excessive liquid He consumption) [15]. New dedicated equipment (cryogenic line, filling tanks, cryocoolers) and instrumentation (gauges) must be implemented leading, thus, to additional costs.

The alternative solution adopted at SOLEIL is to use permanent magnets to produce high energy photons. The technology of permanent magnets, mature for 20 years, offers grades of high magnetizations [16] and coercivities [17]. At PETRA III ( $6 \mathrm{GeV}$ ) and SSRL (3 GeV), a damping wiggler [18] and a multipole wiggler [19] made of permanent magnet and vanadium poles have been built to produce, respectively, a field of 1.5 and $2 \mathrm{~T}$ with periods of 200 and $175 \mathrm{~mm}$. NSLSII ( $3 \mathrm{GeV}$ ) has also designed a damping wiggler of $1.58 \mathrm{~T}$. Table I summarizes the characteristics of moderate field wigglers.

Furthermore, one can push the use of permanent magnets by operating them under vacuum. Indeed, it enables one to reduce the gap between magnet arrays and as a consequence to increase the magnetic field amplitude. The technology of in-vacuum IDs has proven to be very efficient in the production of high field and high number of periods. SPring-8, which initially developed this technology to push photon energy towards $100 \mathrm{keV}$, has built various small gap undulators [20] and one in-vacuum $90 \mathrm{~mm}$ period wiggler [21] producing a $2 \mathrm{~T}$ field at a gap of $7 \mathrm{~mm}$. Other synchrotron facilities, such as Pohang Light Source [22] and Beijing Synchrotron Radiation Facility (BSRF) [23], encouraged by the increase of magnetization value, coercivity and the improvement of the resistance against demagnetization [24], have also opted for the construction of in-vacuum wigglers. In fact, installation of the magnetic system under vacuum makes it possible either to increase the field or the number of periods without producing excessive power. The main idea is that magnetic systems with a large number of radiating poles and a moderate magnetic field are preferable. This strategy has been adopted at SOLEIL through the construction of a wiggler of a period of only $50 \mathrm{~mm}$ and a magnetic field of $2.1 \mathrm{~T}$. As a comparison with the performances of other wigglers mentioned in the Table I, the angular flux of WSV50 has been calculated (Fig. 1) using the dedicated Synchrotron Radiation Workshop (SRW) radiation code [25].

Even if the field is rather moderate, the magnetic attraction between magnet arrays remains large. It depends on the length of the magnetic system and its width. Magnetic forces induce mechanical deformations of girders and the complete frame. $C$-shaped frames commonly used to support the magnetic system are very sensitive to mechanical deformations compared to an $H$-shaped structure but enables a lateral access for magnetic measurements. Instead of increasing the stiffness of girders and the mechanical structure, we proposed on the one hand to reduce the pole

TABLE I. Characteristics of existing and designed moderate field wigglers.

\begin{tabular}{|c|c|c|c|c|c|c|}
\hline Facility & ID name & Technology & Period $[\mathrm{mm}]$ & Field $[\mathrm{T}]$ & Length $[\mathrm{m}]$ & Radiated power $[\mathrm{kW}]$ \\
\hline DIAMOND & SCW60 & Superconducting & 60 & 3.5 & 1.44 & 42 \\
\hline CLS & SCW34 & Superconducting & 34 & 2 & 1.1 & 10.5 \\
\hline PETRA III & DW200 & Out-vacuum permanent magnet & 200 & 1.5 & 40 & 215 \\
\hline SSRL & W175 & Out-vacuum permanent magnet & 175 & 2 & 2.3 & 22 \\
\hline ALBA & W80 & Out-vacuum permanent magnet & 80 & 1.25 & 1 & 3.7 \\
\hline NSLS II & DW100 & Out-vacuum permanent magnet & 100 & 1.58 & 7 & 41.8 \\
\hline PLS & W140 & Out-vacuum permanent magnet & 140 & 2.5 & 2.275 & 34 \\
\hline SPring-8 & W90 & In-vacuum permanent magnet & 90 & 2.1 & 0.9 & 9.5 \\
\hline BSRF & W148 & In-vacuum permanent magnet & 148 & 2 & 2.275 & 21.8 \\
\hline SOLEIL & WSV50 & In-vacuum permanent magnet & 50 & 2.1 & 2 & 21.1 \\
\hline
\end{tabular}




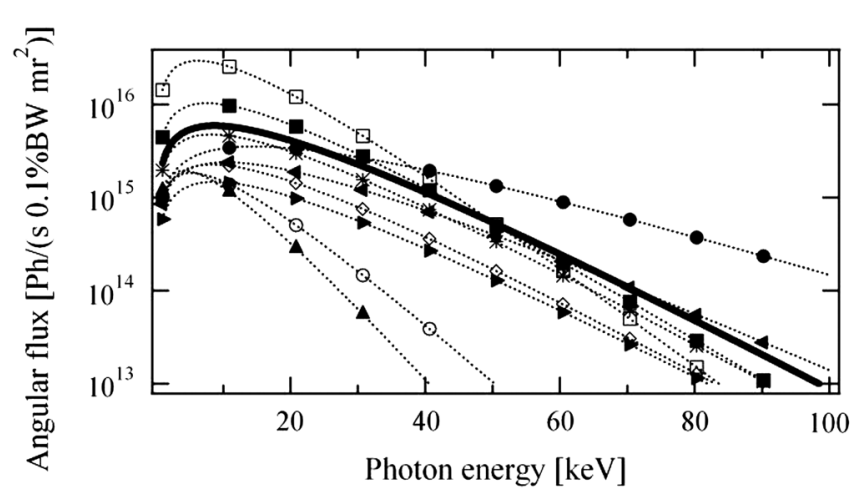

FIG. 1. Angular flux of IDs presented in Table I calculated with SRW at $2.75 \mathrm{GeV}$ : SCW60 (@), SCW34 (*), DW200 ( $\square)$,

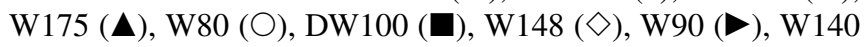
$(\boldsymbol{)})$, and WSV50 ( $)$.

and magnet width and on the other hand to compensate for the magnetic force by a dedicated mechanical system. However, reducing the pole width degrades the transverse homogeneity of the wiggler field. The particles crossing the wiggler experience large kick angles as they travel far from the wiggler axis. This effect is equivalent to the effect of a multipole magnet producing a strong transverse variation of the vertical field integral. This systematic integral is called dynamic field integral (DFI). The effect of the DFI is to reduce the beam lifetime and the injection rate of the electron beam [26]. To overcome the problem, the correction of DFI has been included at the start of the magnetic design phase by the use of cylindrical magnets (magic finger magnets), located at the entrance and exit of the wiggler, producing the opposite field integral.
The design of the wiggler, the counterforce system, magnetic measurements and the correction of the magnetic errors, and the dynamic field integral are presented in this paper.

\section{WIGGLER DESIGN}

Table II presents the characteristics of the wiggler WSV50.

The magnetic design has been performed using the RADIA code [27] and cross-checked with the TOSCA 3D code [28]. Figure 2 shows a reduced scale model of three periods designed with the RADIA code (a) and the expected magnetic field (b). According to both codes, the peak field at the minimum gap $(5.5 \mathrm{~mm})$ reaches $2.1 \mathrm{~T}$ with a difference of $0.3 \%$ between codes linked to the magnet segmentation in the RADIA code and the meshing in the TOSCA 3D code.

The dynamic field integral (DFI) has been calculated from the 2D magnetic field map over a total physical aperture of $5.5 \mathrm{~mm}$ in the vertical and $80 \mathrm{~mm}$ in the horizontal vertical direction [Fig. 3(a)]. In the median plane, a strong peak DFI of $14.5 \times 10^{-4} \mathrm{Tm}$ appears [Fig. 3(b)] at the pole chamfer locations $( \pm 25 \mathrm{~mm})$. To reduce its amplitude, a magnetic system composed of magic finger magnets (Table III) has been designed, built, and installed at the extremities of the ID. The magnets are installed in aluminum holders consisting of 65 holes distributed in three layers. Each hole can receive up to 20 magnets. The residual field integral (addition of 2D DFI map and magic finger magnet map) has been calculated over the same physical aperture [Fig. 3(c)]. In the median plane it has been reduced

TABLE II. Characteristics of the magnetic system of WSV50.

\begin{tabular}{|c|c|c|}
\hline Item & Unit & Value \\
\hline Technology & & Hybrid in-vacuum \\
\hline Magnet grade & & NdFeB (VACODYM 872TP) \\
\hline Magnetization $B_{r}$ & $\mathrm{~T}$ & 1.26 \\
\hline Coercivity $H_{c j}$ & $\mathrm{kA} / \mathrm{m}$ & 2250 \\
\hline Magnet width (horizontal $x$ axis) & $\mathrm{mm}$ & 70 \\
\hline Magnet thickness (longitudinal $s$ axis) & $\mathrm{mm}$ & 17 \\
\hline Magnet height (vertical $z$ axis) & $\mathrm{mm}$ & 50 \\
\hline Magnet chamfer size & $\mathrm{mm} \times \mathrm{mm}$ & $5 \times 5$ (squared shape) \\
\hline Pole material & & Vanadium permendur \\
\hline Pole saturation field $B_{s}$ & $\mathrm{~T}$ & 2.35 \\
\hline Pole width (horizontal $x$ axis) & $\mathrm{mm}$ & 60 \\
\hline Pole thickness (longitudinal $s$ axis) & $\mathrm{mm}$ & 8 \\
\hline Pole height (vertical $z$ axis) & $\mathrm{mm}$ & 40 \\
\hline Pole chamfer size & $\mathrm{mm} \times \mathrm{mm}$ & $5 \times 5\left(45^{\circ}\right.$ shape $)$ \\
\hline Magnetic period $\lambda_{0}$ & $\mathrm{~mm}$ & 50 \\
\hline Number of effective periods & & 38 \\
\hline Length of the magnetic system & $\mathrm{mm}$ & 1992 \\
\hline Gap range & $\mathrm{mm}$ & $5.5-70$ \\
\hline Maximum magnetic field & $\mathrm{T}$ & $2.1 \mathrm{~T}$ \\
\hline
\end{tabular}


(a)
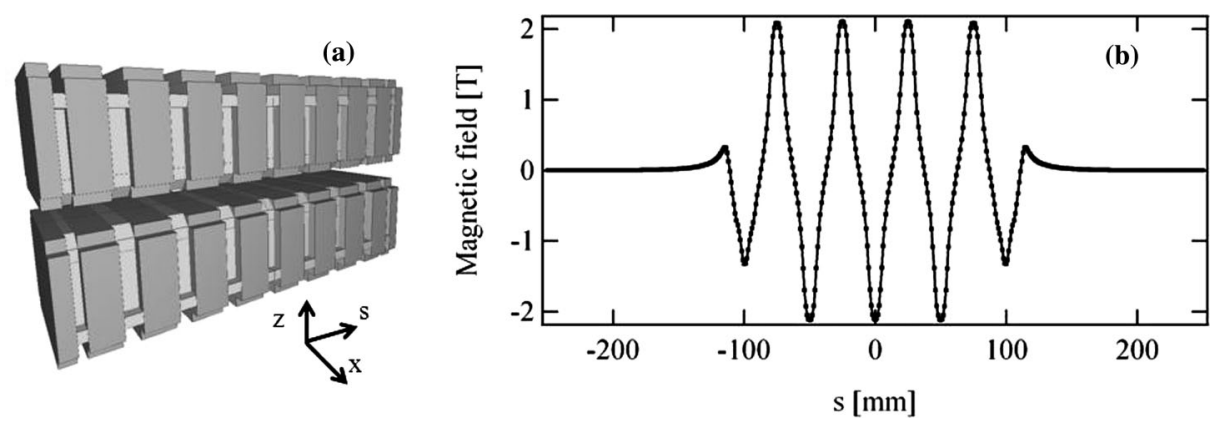

FIG. 2. Three period wiggler (a) designed with RADIA and TOSCA composed of permanent magnets (grey) and vanadium permendur poles (light grey). In (b), calculated magnetic field with RADIA ( - ) and TOSCA 3D ( $\mathbf{\square})$ at minimum gap.
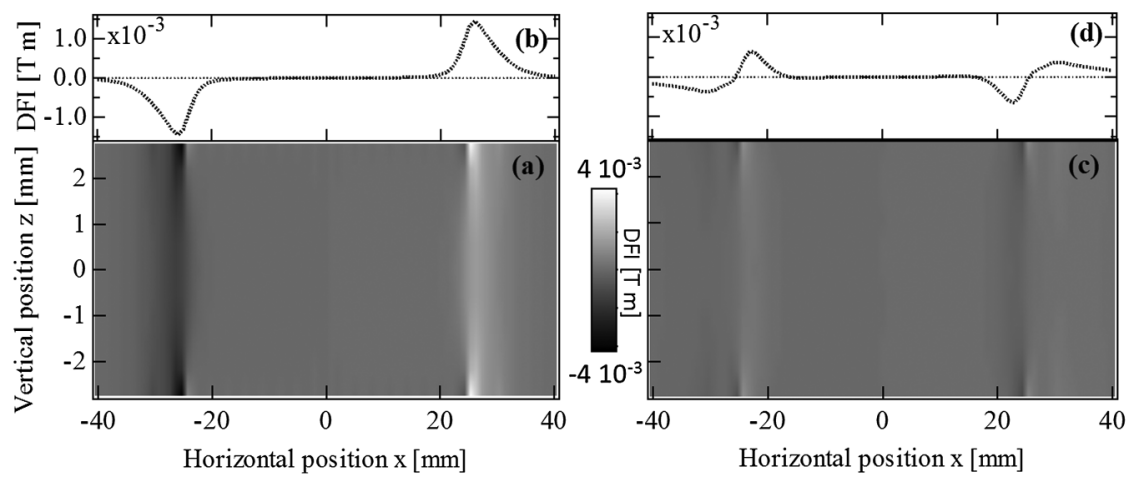

FIG. 3. Calculated 2D DFI (a), median plan $(z=0)$ DFI (b), calculated 2D DFI added to the calculated vertical field 2D map produced by the magic finger magnets $(c)$, median plan $(z=0)$ DFI added to the on-axis vertical field produced by the magic finger magnets (d). Calculations are performed with the RADIA code.

down to $6 \times 10^{-4}$ Tm [Fig. 3(d)]. It was not possible to fully cancel the DFI since the location of the magic finger magnets does not exactly match with the pole chamfer position.

To evaluate the impact of the wiggler on the electron beam dynamics, with and without DFI correction, 2D second order angular kick maps of the wiggler have been generated with the RADIA code, for both the wiggler and the magic finger magnets at minimum gap. The effect of the angular deflections induced by the wiggler and the magic finger magnet system has been analyzed using the TRACYII tracking code [29] in terms of dynamic aperture and energy acceptance [30]. Calculations have been performed with the nominal optics of SOLEIL [31]. Figure 4 shows the effect of the magic finger magnets on the negative energy acceptance. The positive energy acceptance is not modified

TABLE III. Characteristics of magic finger magnets.

\begin{tabular}{lc}
\hline \hline Grade & NdFeB \\
Geometry & Cylindrical shape \\
Length [mm] & 3 \\
Diameter [mm] & 4 \\
Magnetization [T] & 1.2 \\
Magnetization axis & Main cylinder axis \\
\hline \hline
\end{tabular}

by the wiggler because of the limitation imposed, even for the bare machine, by the large value of $\alpha_{2}$ (the second order term of the momentum compaction factor). The wiggler reduces the negative energy acceptance by $0.5 \%$ in long and medium straight sections, then decreasing the

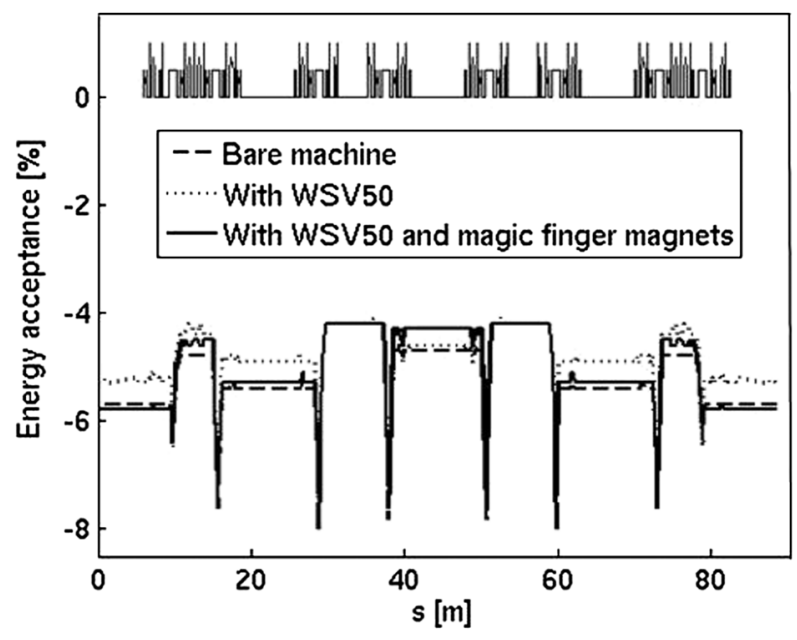

FIG. 4. Calculated variation of the negative energy acceptance along one superperiod (one fourth of the storage ring). Calculations are performed with TRACY II. 
TABLE IV. Pumping capacity of the WSV50.

\begin{tabular}{lcccc}
\hline \hline & & Pumping speed of & Pumping speed of \\
Equipment & Type & $\mathrm{N}_{2}(\mathrm{l} / \mathrm{s}) @ 10^{-6} \mathrm{mbar}$ & $\mathrm{H}_{2}(\mathrm{l} / \mathrm{s}) @ 10^{-9} \mathrm{mbar}$ & Number \\
\hline Ion pump & Varian Vaclon Plus Starcell 150 & 125 & 100 & 4 \\
Ion pump & Varian Vaclon Plus Starcell 500 & 410 & 330 & 1 \\
Ti sublimator & RIAL & 500 & 1000 & 6 \\
NEG cartridge & SAES C2000 DSK & 450 & 2000 & 2 \\
\hline \hline
\end{tabular}

Touschek beam lifetime from 44 to $40 \mathrm{~h}$ (calculated for a $400 \mathrm{~mA}$ beam current stored in 416 bunches). The DFI correction almost restores the negative energy acceptance and a $42 \mathrm{~h}$ Touschek lifetime. Taking into account the $30 \mathrm{~h}$ vacuum beam lifetime, the total beam lifetime of $18 \mathrm{~h}$ should be slightly reduced $(17.5 \mathrm{~h})$ when the wiggler will be closed to minimum gap.

\section{MECHANICAL AND VACUUM DESIGN}

Magnets and poles are mounted on $\mathrm{Al}$ holders which are installed on two stainless steel girders with low magnetic permeability $(<1.06$ after machining). The opening and closing of the gap (5.5 to $100 \mathrm{~mm}$ ) between the magnet arrays are accomplished by two Bergher Lahr VRDM3910 motors. The maximum speed between jaws is $2 \mathrm{~mm} / \mathrm{s}$. The gap is controlled via absolute linear encoders (TR Electronic model) with an accuracy of $0.4 \mu \mathrm{m}$ under load and corrected independently via the motors at the entrance and the exit. A third identical motor installed on the base of the frame allows the whole carriage to be moved vertically over a $10 \mathrm{~mm}$ range to ensure the centering of the wiggler axis with respect to the electron beam axis. The vacuum chamber is composed of one main cylindrical vacuum vessel of $500 \mathrm{~mm}$ diameter and two flanges. The total capacity of pumping is $10730 \mathrm{l} / \mathrm{s}$ distributed among four ion pumps of $125 \mathrm{l} / \mathrm{s}$, one ion pump of 410 l/s, two NEG cartridges, and six titanium sublimation pumps (Table IV). The baking is performed at a maximum temperature of $125^{\circ} \mathrm{C}$ on the magnets for two weeks.

The attraction force $F_{M}$ acting between girders is expressed as

$$
F_{M}=\frac{1}{2 \mu_{0}} \iint B^{2}(x, s) d x d s
$$
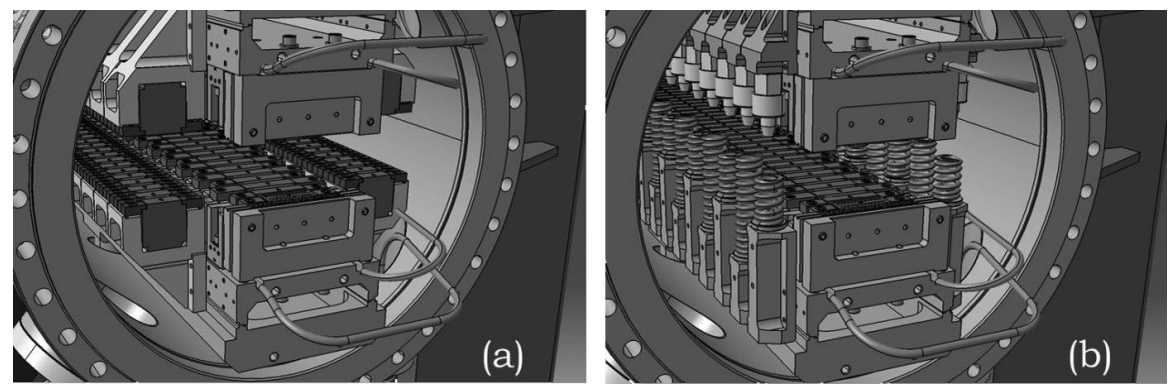

FIG. 5. Magnetic counterforce system (a) and spring counterforce system (b). where $B(x, s)$ is the magnetic field calculated in the median plan $(z=0)$.

The magnetic forces between girders reach 8.5 tons at minimum gap $(4.5 \mathrm{~mm})$. Magnetic attraction of girders can be canceled by using additional permanent magnets with opposite magnetization of the top and bottom girders $[32,33]$. A magnetic system dedicated to the force compensation has been studied first. It is composed of magnets and poles defined in Table II, but the width ( $X$ dimension) is twice lower. The magnetic counterforce system is located on the side of the main ID system [Fig. 5(a)] and is shifted by a quarter of period to limit the magnetic interaction with the main ID system. It completely cancels the magnetic forces whatever the gap is [Fig. 6(a)] but several drawbacks appear. The mutual interaction with the main ID system requires enlarging the girders and as a consequence the vacuum chamber. The full opening of the gap is reduced from $70 \mathrm{~mm}$ down to $50 \mathrm{~mm}$ leading to an increase of the residual radiated power from 2 to $32 \mathrm{~W}$ at maximum gap. By the way, the counterforce system located far from the ID axis $(120 \mathrm{~mm})$ produces a torque of $10 \mathrm{kNm}$ resulting in additional girder deformations of the order of $0.15 \mathrm{~mm}$.

An alternative solution, more compact, has been then proposed and adopted. It consists in replacing the additional magnets by nonmagnetic springs [Fig. 5(b)]. At the first order, the repulsive force of a spring varies linearly with its length. To compensate for the exponential behavior of the magnetic attraction, two types of springs with two different lengths and rigidity coefficient are alternated along the magnetic system. Long springs (LS) and short springs (SS) allow the force to be compensated, respectively, from a gap of $22 \mathrm{~mm}$ down to $4.5 \mathrm{~mm}$ and from $10 \mathrm{~mm}$ down to $4.5 \mathrm{~mm}$. For each gap value the compensation 


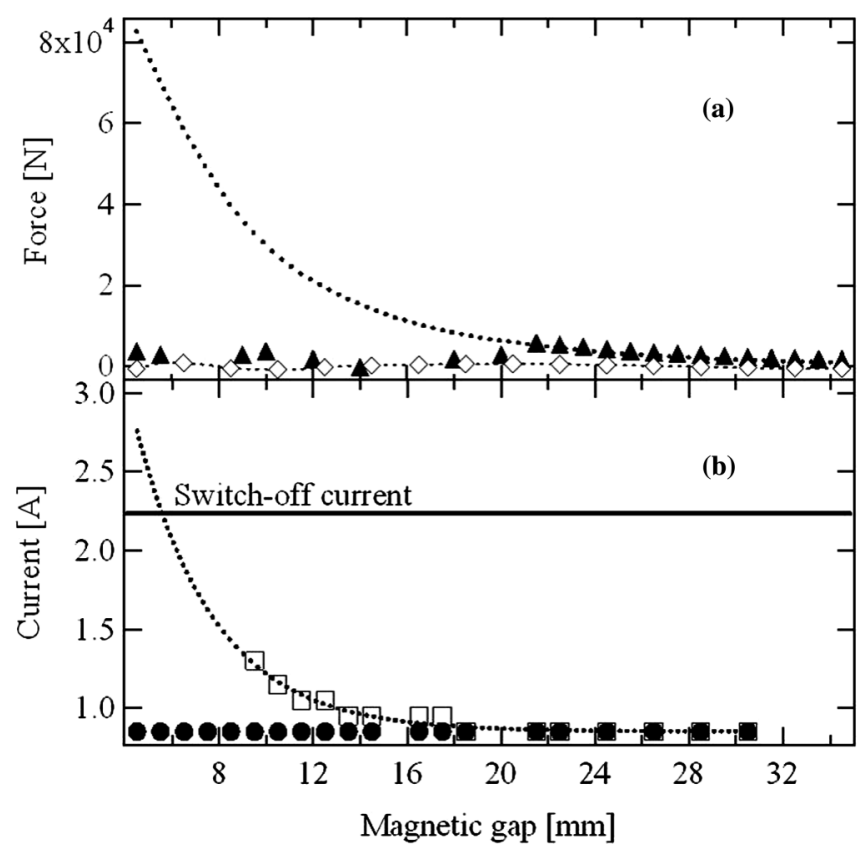

FIG. 6. In (a), calculated force acting on magnet girders without counterforce system (...), with magnetic counterforce system $(\diamond)$, and with spring counterforce system $(\boldsymbol{\Delta})$. In (b), measured current of motors required to move the gap with ( ) or without springs ( $\square$ ).

results from the action of both springs. With this solution, the maximum gap remains $70 \mathrm{~mm}$.

The rigidity coefficient and the length of the springs have been individually measured (Table V) in order to pair them. They have been also equipped with spacers in order to compensate for the length difference between them.

The residual force after installation of the springs is limited to $500 \mathrm{~kg}$ in the gap range of $5.5-70 \mathrm{~mm}$ [Fig. 6(a)]. To check the efficiency of the springs, we have measured the minimum current required to move the gap [Fig. 6(b)] in both conditions when the wiggler is equipped with or without springs. As the magnetic force is attractive, the test is performed by opening the gap. If the ID is equipped with springs, the current remains steady at $0.85 \mathrm{~A}$ (the switch-off limit is $2.25 \mathrm{~A}$ ) during the gap variation. After removing all the springs, the current of motors varies exponentially from 0.85 to 1.25 A during the gap opening.

TABLE V. Characteristics of the springs.

\begin{tabular}{|c|c|c|c|c|}
\hline Type of spring & $\begin{array}{l}\text { Length } \\
{[\mathrm{mm}]}\end{array}$ & $\begin{array}{l}\text { Rigidity } \\
\text { coefficient } \\
{[\mathrm{N} / \mathrm{mm}]}\end{array}$ & Material & $\begin{array}{c}\text { Magnetic } \\
\text { permittivity }\end{array}$ \\
\hline Long spring (LS) & $95 \pm 0.75$ & $53 \pm 0.5$ & InConel & 1.005 \\
\hline Short spring (SS) & $42 \pm 0.5$ & $163 \pm 1$ & InConel & 1.005 \\
\hline
\end{tabular}

TABLE VI. Rigidity coefficient variation of long springs (LS) and short springs (SS) after compression tests. $R$ and $\Delta R$ are, respectively, the mechanical rigidity and its variation after testing.

\begin{tabular}{lrrcc}
\hline \hline Type of spring & $\begin{array}{c}\text { Cycle } \\
\text { number }\end{array}$ & Duration & $\begin{array}{c}R \\
{[\mathrm{~N} / \mathrm{mm}]}\end{array}$ & $\begin{array}{c}\Delta R \\
{[\mathrm{~N} / \mathrm{mm}]}\end{array}$ \\
\hline LS & 1 & $170 \mathrm{~h}$ & 53 & 0.12 \\
& 190 & $2 \mathrm{~h}$ & 53 & 0.08 \\
& 5000 & $26 \mathrm{~s}$ & 53 & 0.05 \\
SS & 1 & $170 \mathrm{~h}$ & 163 & 0.1 \\
& 500 & $5 \mathrm{~s}$ & 163 & 0.05 \\
& 5000 & $22 \mathrm{~s}$ & 163 & 0.03 \\
\hline \hline
\end{tabular}

The rigidity coefficient $R$ of the springs has been checked versus compression rate and the time of compression [34]. The tests have been iterated with a larger number of cycles (5000) representing the total number of gap cycles performed during the lifetime of the wiggler (Table VI). The results show that the rigidity coefficient does not evolve during a long compression time or after a large number of compression cycles.

\section{ASSEMBLING AND MAGNETIC CONTROL}

The measurement bench is equipped with a $7 \mathrm{~m}$ long rotating coil which measures the first integral of the magnetic field. The bench is also equipped with a moving Hall probe system which measures the three components of the magnetic field along the axis of the wiggler. An additional Hall probe (Group3 Teslameter [35]) has been used to calibrate the 3D Hall probes for magnetic field values above $1 \mathrm{~T}$. The three magnetization components of all the magnets, main $\left(B_{r s}\right)$ and parasitic $\left(B_{r x}\right.$ and $\left.B_{r z}\right)$ have been measured (Fig. 7) by means of Helmholtz coils by the manufacturer (Vacuumschmeltze). The main component $\left(B_{r s}\right)$ exceeds the minimum required value $(1.25 \mathrm{~T})$ and the rms dispersion $(0.0025 \mathrm{~T})$ is low in particular because the magnets are magnetized in the direction where the thickness is small.

The measurements of single modules were used for sorting the magnets when assembling the magnetic system. Each single block was equipped with two additional poles to take into account the ferromagnetic effect of them. The horizontal and vertical field integrals $I_{x}$ and $I_{z}$ were measured with the rotating coil versus the horizontal position. The transverse variation of the averaged values $\left\langle I_{x}\right\rangle$ and $\left\langle I_{z}\right\rangle$ over all the modules is presented in Fig. 8. $\left\langle I_{x}\right\rangle$ and $\left\langle I_{z}\right\rangle$ are small on axis $\left(20 \times 10^{-6} \mathrm{Tm}\right)$ which eases the sorting. However, at the magnet block corners $( \pm 25 \mathrm{~mm}),\left\langle I_{z}\right\rangle$ is large $\left(-40 \times 10^{-6}\right.$ and $75 \times 10^{-6} \mathrm{Tm}$, respectively). The peaks of $\left\langle I_{z}\right\rangle$ have been canceled by tilting and displacing horizontally the modules during assembly.

The assembly technique is described in [36]. Following the assembly, the magnetic field was measured along the 

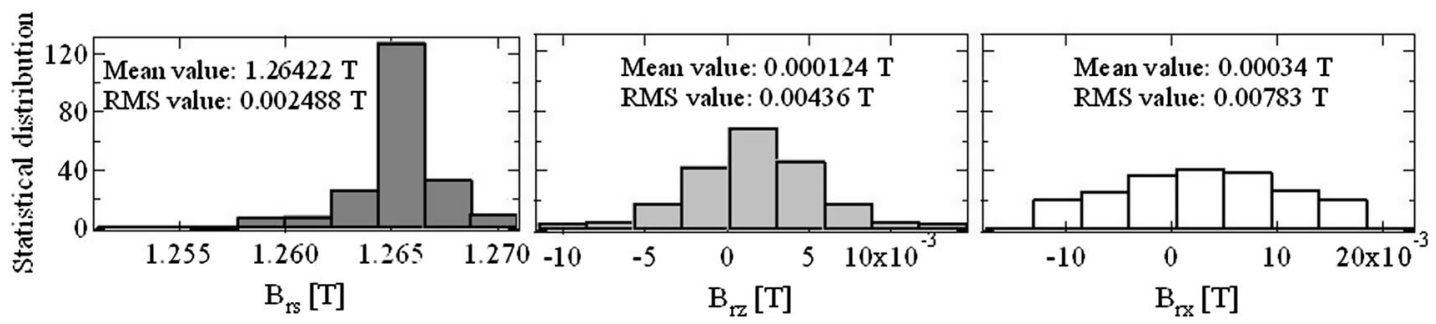

FIG. 7. Magnetization components, main $B_{r s}(\square)$ and parasitic $B_{r z}(\square)$, and $B_{r x}(\square)$ measured with Helmholtz coil.

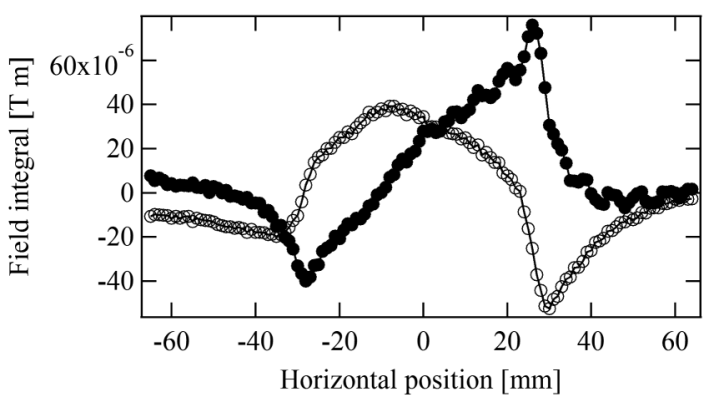

FIG. 8. Averaged measured field integrals $\left\langle I_{x}\right\rangle(\bigcirc)$ and $\left\langle I_{z}\right\rangle$ ( ) with rotating coil.

wiggler axis with the 3D Hall probe system to deduce the harmonic contents (Table VII).

The measured and calculated peak fields agree within $1 \%$. However, the distribution of the harmonics of the measured field differs slightly from those of the calculated field in particular at small gaps $(5.5$ and $7 \mathrm{~mm})$ probably because of a slight saturation at the corner of the poles.

\section{MAGNETIC FIELD CORRECTION}

As the wiggler will operate only at minimum gap, the magnetic correction has been performed only at $5.5 \mathrm{~mm}$ gap. The residual field integrals have been also checked in the vertical and horizontal planes and corrected using magic finger magnets [37]. The correction of the residual field integrals is a converging process based on iterative measurements and predicted magic finger positions. Three iterations have been necessary to correct the field integrals over the required horizontal aperture $( \pm 30 \mathrm{~mm}$ ). At a gap of $5.5 \mathrm{~mm}$, the horizontal and vertical

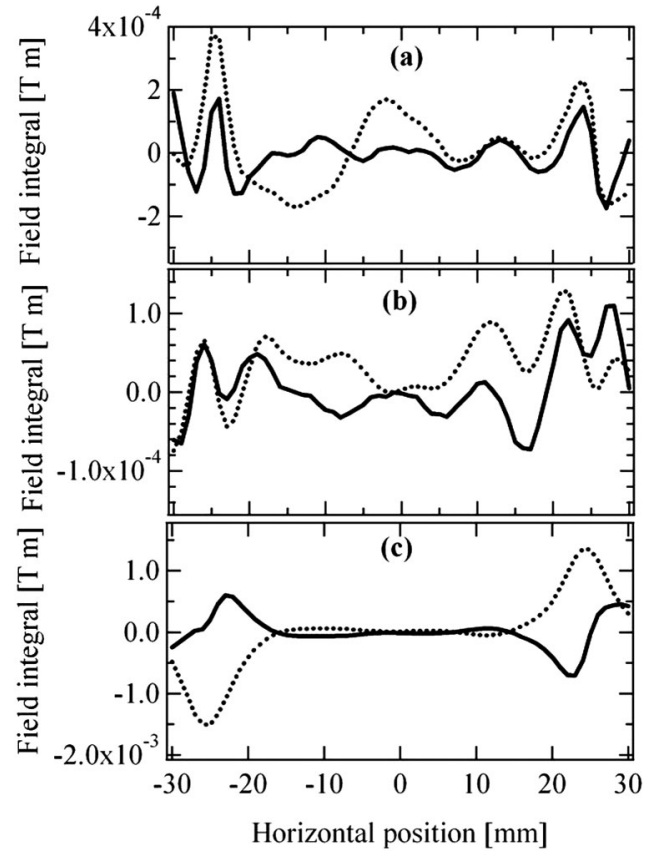

FIG. 9. Multipolar component correction: measured field integral $I_{x}(\mathrm{a}), I_{z}$ (b) at minimum gap $(5.5 \mathrm{~mm})$ before $(.$.$) and after$ $(-)$ use of magic finger magnets. DFI correction: measured $I_{z}$ added to the theoretical DFI (c) at minimum gap $(5.5 \mathrm{~mm})$ before (...) and after ( $)$ use of magic finger magnets.

field integrals remain between $-1.5 \times 10^{-4}$ and $+1.5 \times$ $10^{-4} \mathrm{Tm}$ after correction. The positive effect of magic finger is mainly pronounced in the horizontal plane $\left(I_{x}\right)$. The on-axis field integral has been reduced from $1.1 \times 10^{-4}$ to $10^{-5} \mathrm{Tm}$ and the skew integrated quadrupole component from 0.02 to $0.002 \mathrm{~T}$ [Figs. 9(a) and 9(b)].

TABLE VII. First $\left(B_{1}\right)$, third $\left(B_{3}\right)$, fifth $\left(B_{5}\right)$ Fourier components, average peak field $\left\langle B_{\max }\right\rangle$, and peak field dispersion $\sigma_{B}$.

\begin{tabular}{lcccccccccc}
\hline \hline & \multicolumn{8}{c}{$B(s)=\sum_{n=1}^{n=\infty} B_{n} \cdot \cos \left[2 \pi n s / \lambda_{0}\right]$} \\
Gap [mm $]$ & $\left\langle B_{\max }\right\rangle[\mathrm{T}]$ & $\sigma_{B}[\mathrm{~T}]$ & $B_{1}[\mathrm{~T}]$ & $B_{3}[\mathrm{~T}]$ & $B_{5}[\mathrm{~T}]$ & $\left\langle B_{\max }\right\rangle[\mathrm{T}]$ & $\sigma_{B}[\mathrm{~T}]$ & $B_{1}[\mathrm{~T}]$ & $B_{3}[\mathrm{~T}]$ & $B_{5}[\mathrm{~T}]$ \\
\hline 5.5 & 2.081 & 0.0075 & 1.888 & 0.259 & -0.03 & 2.101 & 0 & 1.83 & 0.32 & -0.008 \\
7 & 1.818 & 0.0069 & 1.701 & 0.19 & -0.023 & 1.842 & 0 & 1.62 & 0.23 & -0.01 \\
10 & 1.313 & 0.0041 & 1.24 & 0.087 & -0.011 & 1.331 & 0 & 1.23 & 0.11 & -0.008 \\
15 & 0.802 & 0.0036 & 0.78 & 0.025 & -0.002 & 0.843 & 0 & 0.81 & 0.03 & -0.002 \\
\hline \hline
\end{tabular}


TABLE VIII. Summary of integrated multipolar components at minimum gap of $5.5 \mathrm{~mm}$.

\begin{tabular}{lrlc}
\hline \hline Gap $[\mathrm{mm}]$ & $Q_{N}[\mathrm{~T}]$ & $Q_{T}[\mathrm{~T}]$ & $S_{N}[\mathrm{~T} / \mathrm{m}]$ \\
\hline 5.5 & 0.00272 & 0.00202 & 0.5691 \\
7 & 0.00084 & 0.00433 & 0.3587 \\
10 & -0.00013 & 0.00647 & 0.1489 \\
15 & 0.00181 & 0.00451 & 0.0708 \\
\hline \hline
\end{tabular}

In a second step, the dynamic field integral (DFI) has been corrected using magic finger magnets. The DFI is the field integrated over the trajectory of electrons crossing the wiggler. Thus, it cannot be measured with the rotating coil. The solution consists in adding the theoretical DFI calculated with RADIA to the vertical field integral measured with the rotating coil and applying a new correction using additional magic finger magnets.

As predicted, the magnitude of the DFI peaks has been strongly reduced from $14.5 \times 10^{-4}$ to $6 \times 10^{-4} \mathrm{Tm}$ [Fig. 9(c)]. The remaining part results from the fact that the magic finger hole positions do not exactly match with the pole chamfer positions. The integrated multipolar components are listed in Table VIII. $Q_{N}, Q_{T}, S_{N}$, respectively the normal quadrupole, skew quadrupole, normal sextupole, are negligible. Thus, no additional focusing, coupling, and chromaticity should be observed on the electron beam.

\section{ON-BEAM TESTS}

\section{A. Tune shift measurements}

The horizontal and vertical tune shifts $\Delta \nu_{x}$ and $\Delta \nu_{z}$ induced by the wiggler result from two effects. First, the natural focusing linked to the amplitude of the field and second, the parasitic focusing coming from the integrated normal gradient (magnetic and mechanical errors). The first contribution in the focusing is written, considering an ID with a vertical periodic magnetic field, as [38]

$$
\begin{gathered}
{\left[\Delta v_{x}\right]_{\text {nat }}=0,} \\
{\left[\Delta v_{z}\right]_{\text {nat }}=\frac{\left\langle\beta_{z}\right\rangle}{4 \pi} \frac{\int_{-\infty}^{+\infty} B_{z}^{2}(s) d s}{(B \rho)^{2}}=\frac{\left\langle\beta_{z}\right\rangle}{8 \pi} \frac{L_{w} \Sigma B_{i}^{2}}{(B \rho)^{2}},}
\end{gathered}
$$

where $\left[\Delta \nu_{x}\right]_{\text {nat }}\left(\left[\Delta \nu_{z}\right]_{\text {nat }}\right)$ is the natural horizontal (vertical) tune shift, $\left\langle\beta_{z}\right\rangle$ the vertical optical function averaged over the length of the wiggler, $(B \rho)$ the electron magnetic rigidity, $B_{i}$ is the $i$ th Fourier component of the magnetic field (see Table VI), $L_{w}$ the length of the wiggler.

The contribution due to field errors is written as [39]

$$
\begin{aligned}
& {\left[\Delta v_{x}\right]_{\mathrm{par}}=\frac{+1}{4 \pi} \frac{\left\langle\beta_{x}\right\rangle}{(B \rho)} \frac{d I_{z}(x)}{d x},} \\
& {\left[\Delta v_{z}\right]_{\mathrm{par}}=\frac{-1}{4 \pi} \frac{\left\langle\beta_{z}\right\rangle}{(B \rho)} \frac{d I_{x}(z)}{d z} .}
\end{aligned}
$$

$\left[\Delta \nu_{x}\right]_{\mathrm{par}}\left(\left[\Delta \nu_{z}\right]_{\mathrm{par}}\right)$ is the parasitic horizontal (vertical) tune shift. $\left\langle\beta_{x}\right\rangle$ is the horizontal optical function averaged over the length of the wiggler. $I_{x}$ and $I_{z}$ are the horizontal and vertical field integrals. It can be noted that due to the Maxwell equations $d I_{x}(z) / d z=d I_{z}(x) / d x$.

The on-axis tune shifts $(x=z=0)$ were calculated from the measured magnetic field and from the integrated harmonic components measured with the rotating coil (Table VIII). The rigidity $B \rho$, the averaged optical functions $\left\langle\beta_{x}\right\rangle$ and $\left\langle\beta_{z}\right\rangle$ equal at SOLEIL respectively $9.13 \mathrm{Tm}$, 14.3 and $2.4 \mathrm{~m}$. The calculated contributions $\left[\Delta \nu_{z}\right]_{\text {nat }}$, $\left[\Delta \nu_{x}\right]_{\mathrm{par}},\left[\Delta \nu_{z}\right]_{\mathrm{par}}$ and the total tune shifts $\Delta \nu_{x}$ and $\Delta \nu_{z}$ are summarized in Table IX. The parasitic contribution is negligible.

Horizontal and vertical tune shifts measured with the electron beam are presented in Fig. 10. The accuracy of the measurements is $\pm 2 \times 10^{-4}$. The agreement with the tune shifts deduced from magnetic measurements is very good.

The vertical focusing of the wiggler destroys the optical function symmetry and the measured beta beat reaches $6 \%$ in the vertical plane. A local correction of the focusing using the ten closest quadrupoles has been implemented in order to restore the optical function symmetry.

\section{B. Field integral measurements}

Any residual magnetic field integral creates at the end of the ID an angular kick $\theta_{x, z}$ which is expressed as

$$
\theta_{x, z}=\frac{1}{(B \rho)} \int_{0}^{L_{w}} B_{z, x}(s) L d s,
$$

where $\theta_{x, z}$ is the angular kick generated, respectively, in the horizontal and vertical plane by the ID. $B_{z, x}$ is the vertical (horizontal) field of the ID. This angular kick impacts the electron horizontal and vertical closed orbits everywhere in the storage ring. The resulting closed orbit distortion $\delta_{x, z}$

TABLE IX. Calculated vertical and horizontal tune shifts $\left[\Delta \nu_{z}\right]_{\mathrm{nat}},\left[\Delta \nu_{x}\right]_{\mathrm{par}},\left[\Delta \nu_{z}\right]_{\mathrm{par}}, \Delta \nu_{x}$, and $\Delta \nu_{z}$ induced by the wiggler versus the gap.

\begin{tabular}{lcrrrr}
\hline \hline Gap $[\mathrm{mm}]$ & {$\left[\Delta \nu_{z}\right]_{\mathrm{nat}}$} & \multicolumn{1}{c}{$\left[\Delta \nu_{x}\right]_{\mathrm{par}}$} & \multicolumn{1}{c}{$\left[\Delta \nu_{z}\right]_{\mathrm{par}}$} & \multicolumn{1}{c}{$\Delta \nu_{x}$} & \multicolumn{1}{c}{$\Delta \nu_{z}$} \\
\hline 5.5 & $7.8 \times 10^{-3}$ & $3.4 \times 10^{-4}$ & $-6 \times 10^{-5}$ & $3.4 \times 10^{-4}$ & $7.8 \times 10^{-3}$ \\
7 & $6.3 \times 10^{-3}$ & $1.0 \times 10^{-4}$ & $-2 \times 10^{-5}$ & $1.0 \times 10^{-4}$ & $6.3 \times 10^{-3}$ \\
10 & $3.3 \times 10^{-3}$ & $-1.5 \times 10^{-5}$ & $2 \times 10^{-6}$ & $-1.5 \times 10^{-5}$ & $3.3 \times 10^{-3}$ \\
15 & $1.3 \times 10^{-3}$ & $2.3 \times 10^{-4}$ & $-4 \times 10^{-5}$ & $2.3 \times 10^{-4}$ & $1.3 \times 10^{-3}$ \\
\hline \hline
\end{tabular}




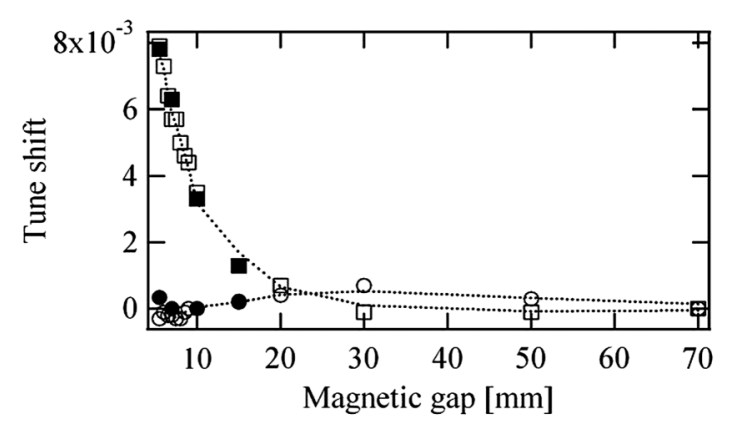

FIG. 10. Tune shift induced by the wiggler versus magnetic gap. Vertical $(\square)$ and horizontal $(\bigcirc)$ tune shifts measured with the electron beam. Vertical $(\boldsymbol{\square})$ and horizontal $(\boldsymbol{O})$ tune shifts deduced from the magnetic measurements.

produced at the location $s_{k}$ respectively in the horizontal $(x)$ and vertical $(z)$ plane is written as follows:

$$
\begin{aligned}
\delta_{x, z}\left(s_{k}\right)= & \frac{\theta_{x, z}(0)}{2}\left[\sqrt{\beta_{x, z}(0) \beta_{x, z}\left(s_{k}\right)}\right. \\
& \times \frac{\cos \left[\phi_{x, z}\left(s_{k}\right)-\phi_{x, z}(0)-\pi \nu_{x, z}\right]}{\sin \left[\pi \nu_{x, z}\right]} \\
& \left.-\frac{2 \eta_{x, z}(0) \eta_{x, z}\left(s_{k}\right)}{C \alpha}\right],
\end{aligned}
$$

where the longitudinal location of the angular kick in the storage ring is assumed to be at $s=0 . \beta_{x, z}(0)$ and $\beta_{x, z}\left(s_{k}\right)$ are the horizontal $(x)$ and vertical $(z)$ betatron functions at the location of the kick $s=0$ and observation point $s_{k}$, respectively. $\nu_{x, z}$ are the horizontal $(x)$ and vertical $(z)$ betatron tunes, respectively. $\eta_{x, z}(0)$ and $\eta_{x, z}\left(s_{k}\right)$ are the horizontal $(x)$ and vertical $(z)$ dispersion functions at the location of the kick $(s=0)$ and observation point $\left(s_{k}\right)$, respectively. $C$ is the circumference of the storage ring $(354.097 \mathrm{~m})$ and $\alpha$ the momentum compaction factor $\left(4.5 \times 10^{-4}\right), \phi_{x, z}(0)$ and $\phi_{x, z}\left(s_{k}\right)$ are the betatron phase advances in the horizontal $(x)$ and vertical $(z)$ plane at the angular kick location $(s=0)$ and the observation point location $\left(s_{k}\right)$, respectively. $\phi_{x, z}\left(s_{k}\right)$ is defined as

$$
\phi_{x, z}\left(s_{k}\right)=\int_{0}^{s_{k}} \frac{1}{\beta_{x, z}(s)} d s .
$$

Since the total length of the closed orbit remains constant and is determined by the frequency of the rf system, the particle energy is changed in order to compensate for the orbit lengthening. The term in the bracket which is proportional to $\eta_{x, z}(0) \eta_{x, z}\left(s_{k}\right)$ in Eq. (11) is related to this energy deviation [40,41].

The evaluation of the field integrals is based on the closed orbit distortion (COD) recorded at the location of 120 electron beam position monitors, on the theoretical optical functions $\beta_{x, z}, \eta_{x, z}$, and $\nu_{x, z}$ calculated with TRACYII code and by using Eqs. (6)-(8). This method is applied for on-axis field integral and also for off-axis field integral measurements when generating orbit displacements of the

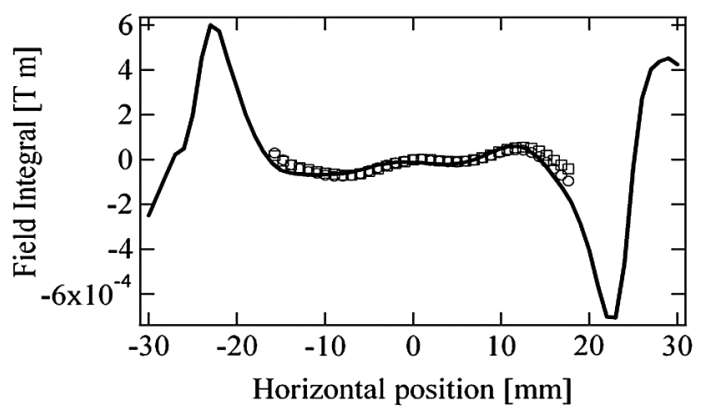

FIG. 11. Transverse variation of the vertical field integrals measured with rotating coils ( $(-)$ ), deduced from closed orbit distortion ( $\square$ ) and from horizontal tune shift $(\bigcirc)$.

electron beam inside the wiggler by means of the storage ring dipolar correctors. In addition, the measurement of the horizontal tune shift $\Delta \nu_{x}$ induced by the wiggler versus the horizontal electron beam displacement and the integration of Eq. (10) make it possible to confirm the values of the off-axis vertical integrals $I_{z}$. Figure 11 shows with a very good agreement the transverse variation of the vertical field integral according to the rotating coil measurements compared to COD and tune shift measurements.

\section{Injection rate and beam lifetime}

The first time the wiggler has been closed to minimum gap, the storage ring was operating with the nominal optics [30]. As predicted by calculations, the injection efficiency was not strongly modified (only from 95\% to 90\%). Contrary to calculations, the total beam lifetime was impacted significantly with a decrease of $3 \mathrm{~h}$ over the $20 \mathrm{~h}$ nominal value. Presently the storage ring is operating with a new optics [42]. To check the impact of the wiggler on the electron beam dynamics, systematic measurements of emittance, injection rate, and beam lifetime have been performed as a function of the gap. Because of its high field, the effect of the wiggler on the horizontal emittance is not negligible as predicted by calculations. The effect of the wiggler on the injection rate is confirmed to be negligible at minimum gap: the nominal efficiency of $90 \%$ is reduced to $85 \%$. However, the total beam lifetime $\tau$ (for a $400 \mathrm{~mA}$ electron beam stored current) is reduced from $15 \mathrm{~h}$ at maximum gap (bare machine) to $11 \mathrm{~h}$ at minimum gap (Table X).

Notice that the physical aperture of the storage ring is routinely set, using two vertical scrapers, to a value smaller than the wiggler gap, in order to protect magnets from particle losses. The beam lifetime cannot be affected by the reduction of the wiggler vertical aperture at minimum gap and the reduction of the beam lifetime is due to nonlinear effects introduced by the DFI. This means that the correction of the dynamic field integral peak from $14.5 \times 10^{-4} \mathrm{Tm}$ down to $6 \times 10^{-4} \mathrm{Tm}$ is not sufficient to cancel the effect of the wiggler on electron beam dynamics. As a consequence, it is foreseen to further reduce 
TABLE X. Variation of horizontal emittance $\varepsilon_{x}$, injection rate $R$, and beam lifetime $\tau$ versus gap.

\begin{tabular}{lcccc}
\hline \hline $\begin{array}{l}\text { Gap } \\
{[\mathrm{mm}]}\end{array}$ & $\begin{array}{c}\text { Measured } \\
\Delta \varepsilon_{x} / \varepsilon_{x}[\%]\end{array}$ & $\begin{array}{c}\text { Expected } \\
\Delta \varepsilon_{x} / \varepsilon_{x}[\%]\end{array}$ & $\begin{array}{c}\text { Measured } \\
R[\%]\end{array}$ & $\begin{array}{c}\text { Measured } \\
\tau[\mathrm{h}]\end{array}$ \\
\hline 70 & 0 & 0 & 90 & 15.2 \\
15 & -0.1 & -0.07 & 89 & 15 \\
11 & -0.1 & 0.6 & 88 & 14.7 \\
5.5 & 2.6 & 3.6 & 85 & 11.2 \\
\hline \hline
\end{tabular}

the DFI peaks using additional magic finger magnets. Nonetheless, the beam lifetime reduction is acceptable and the storage ring can operate in top-up injection mode. The wiggler is presently used routinely at minimum gap by the photon beam line scientists.

\section{CONCLUSION}

WSV50 is a compact in-vacuum wiggler which produces intense flux in the hard $x$-ray domain. A large number of periods was preferred over a high magnetic field because it would generate useful flux rather than excessive power. Even if the ID operates at moderate field, the magnetic attraction reaches almost 10 tons. A counterforce system that reduces magnetic force down to $500 \mathrm{~kg}$ has been designed, built, installed in the magnetic system, and successfully tested. The compensation of the dynamic field integral has also been implemented on the magnetic system from the beginning of the construction in order to maintain a high injection rate and an acceptable beam lifetime during operation of the wiggler. The wiggler is routinely operating on the storage ring at $5.5 \mathrm{~mm}$ gap for beam line experiments. Operation at lower gap $(4.5 \mathrm{~mm})$ is being studied as a means of increasing the flux of photons at high energy. In addition, the recent experimental results on magnets operating at cryogenic temperatures are very promising [43-46] in terms of gain in magnetic field. This option is in phase of discussion to further increase the flux at high photon energy.

\section{ACKNOWLEDGMENTS}

The authors would like to thank Dr. O. Chubar who shared his experience in the optimization of spectral and magnetic performances of IDs, the Survey Group, the Vacuum Group and Engineering Group of SOLEIL for their contribution in the design and construction of the wiggler.

[1] H. Motz, W. Thon, and R. N. Whitehurst, J. Appl. Phys. 24, 826 (1953).

[2] C.S. Hwang, Phys. Rev. ST Accel. Beams 14, 040801 (2011).
[3] N. A. Vinokurov, Phys. Rev. ST Accel. Beams 14, 040701 (2011).

[4] P. Elleaume and H. Onuki, Undulators, Wigglers and Their Applications (Orbiting Books, United Kingdom, 2003), ISBN: 9780415280402.

[5] D. Berger, E. Weihreter, N. Mezentsev, and V. Shkaruba, in Proceedings of the 8th European Particle Accelerator Conference, Paris, 2002 (EPS-IGA and CERN, Geneva, 2002), pp. 2595-2597.

[6] V. Korchuganov, N. Mezentzev, and A. Valentinov, in Proceedings of SRI2006 (American Institute of Physics, Daegu, 2006), pp. 440-443.

[7] K. J. Kim, Nucl. Instrum. Methods Phys. Res., Sect. A 246, 67 (1986).

[8] L. Smith, Lawrence Berkeley Laboratory Internal Report No. LBL21391.

[9] R. Walker, Nucl. Instrum. Methods Phys. Res., Sect. A 237, 366 (1985).

[10] S. Khrushchev, V. Lev, N. Mezentsev, E. Miginsky, V. Repkov, V. Shkaruba, V. Syrovatin, and V. Tsukanov, in Proceedings of RuPAC, Novosibirsk, 2006, p. 398 [http:// www.jacow.org/].

[11] L. Tosi, C. Knapic, and D. Zangrando, in Proceedings of the 9th European Particle Accelerator Conference, Lucerne, 2004 (EPS-AG, Lucerne, 2004) , p. 390.

[12] E. A. Bekhtenev, S. V. Khruschev, E. A. Kuper, V. H. Lev, N. A. Mezentzev, E. G. Miginsky, V. V. Repkov, B. A. Shkaruba V. M. Syrovatin, and V.M. Tsukanov, Phys. Part. Nucl. Lett. 3, 516 (2006), Suppl. 1.

[13] L. Tosi et al., in Proceedings of the 9th European Particle Accelerator Conference, Lucerne, 2004 (Ref. [11]), p. 390.

[14] C. Boffo et al., in Proceedings of the 32nd Free Electron Laser Conference, Malmö, Sweden (Max-lab, Sweden, 2010), p. 660.

[15] E. C. M. Rial and J.C. Schouten, in Proceedings of the International Particle Accelerator Conference, Kyoto, Japan (ICR, Kyoto, 2010), p. 3195.

[16] http://www.vacuumschmelze.de.

[17] http://www.hitachi-metals.co.jp.

[18] M. Tischer, K. Balewski, A. Batrakov, I. Ilyin, D. Shichkov, A. Utkin, P. Vagin, and P. Vobly, in Proceedings of the 11th European Particle Accelerator Conference, Genoa, 2008 (EPS-AG, Genoa, Italy, 2008), pp. 2317-2319.

[19] http://www-ssrl.slac.stanford.edu/.

[20] T. Hara, T. Tanaka, T. Tanabe, X.-M. Maréchal, and H. Kitamura, J. Synchrotron Radiat. 5, 403 (1998).

[21] X. M. Marechal, T. Bizen, T. Hara, T. Seike, T. Tanake, and H. Kitamura, Nucl. Instrum. Methods Phys. Res., Sect. A 467, 138 (2001), Part 1.

[22] D. E. Kim, H. G. Lee, H. S. Suh, H. S. Han, Y. G. Jung, K. H. Park, and C. W. Chung, in Proceedings of SRI2006 (Ref. [6]), pp. 331-334.

[23] C. Shi, Y. Jing, D. Li, Q. Peng, H. Pan, and S. Sheng, "Design and Construction of the First In-Vacuum Wiggler for BSRF," in Proceedings of APAC Conference, 2004, pp. 353-355 [http://www.jacow.org/].

[24] T. Bizen, Y. Asano, T. Hara, X. Marechal, T. Seike, T. Tanaka, H. S. Lee, D.E. Kim, C. W. Chung, and H. Kitamura, Nucl. Instrum. Methods Phys. Res., Sect. A 515, 850 (2003). 
[25] O. Chubar and P. Elleaume, "Accurate and Efficient Computation of Synchrotron Radiation in the Near Field Region," in Proceedings of the EPAC Conference, 1998, pp. 1177-1179 [http://www.jacow.org/].

[26] J. Safranek, C. Limborg, A. Terebilo, P. Elleaume, and K. I. Blomqvist, Phys. Rev. ST Accel. Beams 5, 010701 (2002).

[27] RADIA is a collaboration ESRF/SOLEIL and is freely available for download from http://www.esrf.eu/ Accelerators/Groups/InsertionDevices/Software/Radia.

Details have been published in: P. Elleaume, O. Chubar, and J. Chavanne, in Proceedings of the Particle Accelerator Conference, Vancouver, BC, Canada, 1997 (IEEE, New York, 1997).

[28] OPERA-3D, TOSCA, Vector Fields Ltd., Oxford, United Kingdom.

[29] TRACYII: “A 6D true sympletic tracking code," SOLEIL version.

[30] M.-P. Level et al., in Proceedings of the 8th European Particle Accelerator Conference, Paris, 2002 (Ref. [5]), pp. 212-214.

[31] O. Marcouillé, P. Berteaud, P. Brunelle, N. Béchu, L. Chapuis, M.-E. Couprie, J.-M. Filhol, C. Herbeaux, A. Lestrade, J. L. Marlats, A. Mary, M. Massal, M.-H. Nguyen, K. Tavakoli, M. Valléau, and J. Vétéran, in Proceedings of the International Particle Accelerator Conference, Kyoto, Japan (Ref. [15]), pp. 3102-3104.

[32] T. Bizen, T. Hara, T. Tanaka, X.-M. Marechal, T. Seike, and H. Kitamura, in Proceedings of SRI Conference, San Francisco, 2003 (American Institute of Physics, New York, 2003), pp. 175-178.

[33] R. Carr, J. Synchrotron Radiat. 10, 269 (2003).

[34] O. Marcouille, P. Brunelle, O. Chubar, M.-E. Couprie, J.-M. Filhol, C. Herbeaux, J.-L. Marlats, A. Mary, and K. Tavakoli, in Proceedings of the 11th European Particle Accelerator Conference, Genoa, 2008 (Ref. [18]), pp. 2288-2290.

[35] Group3 Teslameter: http://www.danfysik.com.
[36] O. Chubar, O. Rudenko, C. Benabderrahmane, F. Briquez, M.E. Couprie, O. Marcouille, F. Marteau, and J. M. Filhol, in Proceedings of SRI2006 (Ref. [6]), pp. 359-362.

[37] O. Marcouille, P. Brunelle, L. Chapuis, M.-E. Couprie, J.-M. Filhol, C. Herbeaux, A. Lestrade, J.-L. Marlats, M. Massal, A. Mary, M.-H. Nguyen, K. Tavakoli, M. Valleau, and J. Veteran, in Proceedings of the SRI Conference (American Institute of Physics, Melbourne, 2009), pp. 556-559.

[38] H. Wiedemann, Particle Accelerator Physics I and II (Springer, New York, 1994).

[39] P. Brunelle, Part. Accel. 39, 89 (1992).

[40] F. Amman, Laboratori Nazionali di Frascati Report No. LNF-71/82, 1971.

[41] V. E. Blinov, A. V. Bogomyagkov, V. A. Kiselev, S. A. Nikitin, I. Ya. Protopopov, K. Yu. Todyshev, and G. M. Tumaikin, Nucl. Instrum. Methods Phys. Res., Sect. A 494, 68 (2002).

[42] A. Loulergue, C. Benabderrahmane, F. Bouvet, P. Brunelle, M.E. Couprie, J.C. Denard, T. Moreno, A. Nadji, L. Nadolski, and F. Polack, in Proceedings of the International Particle Accelerator Conference, Kyoto, Japan (Ref. [15]), pp. 2496-2498.

[43] T. Hara, T. Tanaka, H. Kitamura, T. Bizen, X. Maréchal, T. Seike, T. Kohda, and Y. Matsuura, Phys. Rev. ST Accel. Beams 7, 050702 (2004).

[44] J. Chavanne, M. Hahn, R. Kersevan, C. A. Kitegi, C. Penel, and F. Revol, in Proceedings of the 11th European Particle Accelerator Conference, Genoa, 2008 (Ref. [18]), pp. 2243-2245.

[45] C. Benabderrahmane, M. Valléau, P. Berteaud, K. Tavakoli, J.L. Marlats, R. Nagaoka, N. Béchu, D. Zerbib, P. Brunelle, L. Chapuis, D. Dallé, C. Herbeaux, A. Lestrade, M. Louvet, and M. E. Couprie, Nucl. Instrum. Methods Phys. Res., Sect. A 669, 1 (2012).

[46] J. Bahrdt et al., in Proceedings of the International Particle Accelerator Conference, Kyoto, Japan (Ref. [15]), pp. 3111-3113. 